\title{
Honoring our helpers
}

\author{
Roland Talanow $^{1 *}$, Frederik Giesel $^{2}$ \\ 1. EduRad, Lake Tahoe, NV, USA \\ 2. Department of Radiology, University of Heidelberg, Heidelberg, Germany \\ * Correspondence: Dr. Roland Talanow, EduRad, 297 Kingsbury Grade, Suite 100, Mail Box 4470, \\ Lake Tahoe (Stateline), NV 89449-4470, USA \\ (-4 roland@talanow.net) \\ Radiology Case. 2021 Jan; 15(1):30-32 :: $\quad$ DOI: $10.3941 /$ jrcr.v15i1.4273
}

\begin{abstract}
This special issue of the Journal of Radiology Case Reports honors the reviewers who donated their time and expertise throughout the year 2020 to the high quality and success of this journal.
\end{abstract}

\section{EDITORIAL}

This special issue of the Journal of Radiology Case Reports is dedicated to the people who contributed a significant amount to the high quality and success of this journal - our reviewers. Peer-review ensures high scientific and academic quality and accuracy. Reviewers work "behind the scenes" and provide their expertise on a volunteer basis, which deserves a special honor. Their comments are highly appreciated by the editorial team, the authors and of course in the end by the readers. Since 2009 the Journal of Radiology Case Reports implemented a reviewer evaluation system and gives each reviewer a specific score based on the quality of the review. Reviewers with a score of at least 3.5 (score range goes from 1 to 5) are awarded in our online "Hall of Fame" [1] as well as in our yearly thank you note. Detailed information can be found at our guideline page [2]. We would like to thank the following people for their donation of time and expertise in the year 2020:
Abdi, Mohammad

Abdul rahim, Ezamin

Ahmad, Zohra

Ahmadli, Uzeyir

Ahmed, Zahoor

Al-mallah, Dr. adel

Al-shatouri, Mohammad

Alagbe, Olayemi

Alahmari, Abdulwahab

Arora, Jyoti

Ashraf, Junaid

Atwal, Swapndeep

Aydin, Sonay

Ayouche, Othman

Bagade, Swapnil

Bailey, Katie

Bannur, Mahesh

Behairy, Noha

Bhagat, Swapnil

Bhargava, Peeyush

Bhaya, Anil 
Boffano, Michele

Brar, Rahat

Campioni, Paolo

Carraro do nascimento, Vinicius

Chamarthy, Murthy

Chang, Warren

Chauhan, Ankita

Chaurasia, Akhilanand

Chidambaranathan, Natesan

Cossu, Alberto

D'ortenzio, Robert

Das, Joe

De leucio, Alessandro

Degheili, Jad

Dehghan, Pooneh

Desai, Paresh

Di stasi, Martina

Doweidar, Ahmed

Drabkin, Michael

Eichhorn, Joshua

Eksi, Murat sakir

El homsi, Maria

Elmokadem, Ali

Eser, Mehmet bilgin

Espinosa leal, Francisco

Fukuda, Takeshi

G, Padmapriya

Gandhi, Darshan

Gautam, Rupesh

Gehani, Anisha

Gonzalez, Marta

Goyal, Pradeep

Greco, Fabio

Gupta, Amit

Gupta, Priyank

Gupta, Khushboo

Gurdal kosem, Esra

Hamirani, Yasmin

Hegde, Shilpa

Helmy, Daniel

Hesam shariati, Mohammad bakhtiar

Ho, Chang
Hoang, Vantrung

Hongsakul, Keerati

Hoodeshenas, Safa

Hoots, Glenn

Hui, Peter

Izadi, Meisam

Jensen-kondering, Ulf

Kachewar, Sushil

Karatay, Emrah

Kaya, Diana

Kraniotis, Pantelis

Kujak, Jennifer

Kumar, Dinesh

Kumar, Devendra

Landolsi, Manel

Lee, Matthew

Lenobel, Scott

Leung, Vincent

Mamere, Augusto

Mateus marques, Rui

Mcgill, Kevin

Mehra, Shibani

Michimoto, Kenkichi

Mittal, Somit

Mody, Priya

Mohamed, Rania

Momeni, Mohammad

Morelli, Umberto

Mostafa, Mai

Murmura, Elena

Mustafa, Syed abdur rahman

Neupane, Nirmal

Nezami, Nariman

Nguyen, Kevin

Nirwan, Lalit

Ozturk, Kerem

Palmucci, Stefano

Parry, Arshed

Patil, Aruna

Puthussery, Paul

Raouf, Sameh

Rezaeian, Nahid 
Roda, Manohar

Roh, Simon

Sadeghi, Akram

Sarma, Sanjeeb

Schoenhagen, Paul

Senger, Krishna pratap singh

Shankar, Sridhar

Shebel, Haytham

Singh, Gurvinder

Singh, Charanjeet

Sleeba, Teena

Stuppner, Sigmund

Tappouni, Rafel

Vijayaraghavan, Gopal

Wani, Abdul haseeb

Weiss, Erik

Wexler, Lewis

Wortsman, Ximena

Thank you!

Roland Talanow, MD, PhD - Editor-in-Chief

Frederik Giesel, MD, MPh - Associate Editor

\section{REFERENCES}

1. Journal of Radiology Case Reports Hall of Fame. URL: http://www.radiologycases.com/index.php/radiologycases/p ages/view/Radiology-Case-Reports-Hall-of-Fame

2. Journal of Radiology Case Reports Editorial Policies Peer Review Process. URL: http://www.radiologycases.com/index.php/radiologycases/a bout/editeditorialPoli\#peerReviewProcess

\section{Online access}

This article is online available at: www.radiologycases.com/index.php/radiologycases/article/view/4273

\section{Peer discussion}

Questions? Discuss any journal related topic in our protected discussion forum at: www.radiolopolis.com/forums/JRCR

Published by EduRad

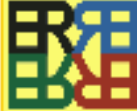

www.EduRad.org 